\title{
Diseño de una Alternativa de Abastecimiento, Canales Abiertos y Estructuras Hidráulicas en el Riego por Superficie de la Granja Experimental de la Universidad Surcolombiana
}

\section{Design of an Alternative for Supply, Open Channel Irrigation and Hydraulic Structures in the Area of the Experimental Farm South Colombian University.}

Jaime Izquierdo Bautista ${ }^{1}$, Edinson Mujica Rodríguez ${ }^{2}$ y Damaris Perdomo Medina ${ }^{3}$.

\section{Resumen}

La granja de la Universidad presenta actualmente canales abiertos en tierra en malas condiciones, canales con formas irregulares ocasionadas por la erosión, la falta de mantenimiento, el uso indiscriminado del agua en la aplicación del riego por superficie, factores que le resta eficiencia al sistema de riego. Es por esto, que surgió la necesidad de hacer este proyecto que se basó fundamentalmente en el trazado y diseño de la red de canales de la granja, proponiendo tres opciones de revestimiento con estructuras hidráulicas que permitan aprovechar mejor el recurso hídrico.

Se propuso una nueva línea de abastecimiento, se realizó entonces el estudio de las posibilidades de captación del agua, quedando finalmente el diseño desde el canal lateral 4 OPIA del distrito de riego ASOJUNCAL, en su parte final, captando 30 l/s de agua, por una conducción en tubería enterrada con una pendiente de uno por mil $(0.1 \%)$.

Palabras clave: riego por superficie; canal abierto; Abastecimiento.

\begin{abstract}
The farm of the University currently has open channels on the ground in poor conditions, irregularly shaped channels caused by erosion, lack of maintenance, the indiscriminate use of water and the inappropriate application of surface irrigation, which reduces efficiency of the system. Because of this, it became necessary to create this project which relies heavily on the layout and design of the network of farm channels, , propose three options for coating and design of hydraulic structures to increase irrigation efficiency.
\end{abstract}

Then we realized the design of a new supply line coming from the channel OPIA ASOJUNCAL irrigation district, at the end of this, capturing $301 / \mathrm{s}$ of water.

Key Words: irrigation by surface; open channel; Supplying. 


\section{Introducción}

Los sistemas agrícolas de producción y especialmente la relacionada con el cultivo de arroz, demandan altos volúmenes de agua que hacen insostenibles esta actividad. Por lo anterior, se requieren mayores esfuerzos en investigación dirigida a hacer más eficiente la producción agrícola con riego mediante el empleo de láminas estrictamente necesarias en el beneficio del cultivo, evitándose daños a la plantación, a los suelos y como consecuencia mayores costos en producción.

En la actualidad se deberían estar utilizando métodos de riego cuantificados en donde se controlen los tiempos de riego en función del volumen de agua a aplicar, para proteger y conservar el recurso hídrico y los suelos agrícolas. Es por esta razón que las estructuras hidráulicas de aforo y de reparto son tan necesarias para ayudar a cuantificar el agua que ingresa a la granja y que se distribuye en el lote para no seguir realizando el riego corrido incontrolado donde se desperdician grandes volúmenes de agua.(El riego, 2009).

La eficiencia con que se aplica el agua de riego al suelo depende de dos factores fundamentales: las características hidrodinámicas del suelo, que se pueden mejorar pero tarda y cuesta mucho, y el manejo de agua durante el riego. En el manejo del agua se distinguen varios aspectos que interactúan e inciden en forma determinante en la eficiencia de aplicación del agua de riego: el diseño del sistema de riego, los caudales utilizados, la frecuencia y el tiempo de riego.

El riego por superficie es el método de riego menos costoso en instalación y mantenimiento, una vez que el agua llega a la parcela no existe coste en la aplicación del agua. Pero, es sin embargo el sistema de riego que utiliza el agua de forma menos eficiente, aun cuando se realiza un adecuado diseño y manejo del riego.

Como se puede apreciar, actualmente son muy pocos los avances de ingeniería que se realizan en este campo comparado con el riego a presión. Lo irónico es, que aunque este método es mucho más económico para la instalación y operación no se invierte en corregir los problemas que durante la historia que lleva se vienen presentando por su utilización. Para que sea aún más económico para el agricultor en este caso a los arroceros utilizar este método de riego.

Según el artículo Canal de riego: Dentro del diseño de riego, los canales de riego tienen la función de conducir el agua desde la captación hasta el campo o huerta donde será aplicado a los cultivos. Son obras de ingeniería importantes, que deben ser cuidadosamente pensadas para no provocar daños al ambiente y para que se gaste la menor cantidad de agua posible. Están estrechamente vinculados a las características del terreno, generalmente siguen aproximadamente las curvas de nivel de este, descendiendo suavemente hacia cotas más bajas (dándole una pendiente descendente, para que el agua fluya más rápidamente y se gaste menos líquido).

Según el artículo el flujo en canales abiertos y su clasificación: El trapecio es la forma más común para canales con bancas en tierra sin recubrimiento, debido a que proveen las pendientes necesarias para la estabilidad.

Según Ojeda (1995), La velocidad del flujo en el canal está limitada por la sedimentación y la erosión. Por lo tanto, la velocidad que se escoja, no debería producir ninguno de estos dos fenómenos. Pero, lograr esto en muchos casos no es posible. La velocidad está limitada entonces por el tipo de revestimiento que tiene el canal, la rugosidad de este y la pendiente adecuada para que ni se erosione ni se sedimente.

Dentro de las estructuras que se necesitan en los canales, el aforador Parshall Según Pedroza, (1995) es una estructura hidráulica que permite medir la cantidad de agua que pasa por una sección de un canal. Consta de cuatro partes principales: Transición de entrada, Sección convergente, Garganta, Sección divergente.

Según el artículo Medición de caudales: La Canaleta Ballofet es otra estructura de aforo que pertenece a los aforadores de flujo crítico. Este se caracteriza por tener paredes paralelas y fondo plano, por lo cual se hace extremadamente fácil su construcción, a la vez posee características de solidez y resistencia a las condiciones de campo. Además, si se presenta escurrimiento critico en la garganta, no se ve afectada por problemas de sedimentación. (Medición 2009).

Las canaletas WSC (Washington State College) es otro aforador de profundidad crítica de un diseño similar al Parshall, que resulta particularmente útil como aforador portátil para mediciones eventuales de pequeños caudales en corrientes o canales sin revestir (Chamberlain 1952). 
Dentro de los tipos de recubrimiento, El suelo-cemento es una opción que consiste en la mezcla íntima de suelo con cemento compactado en su contenido óptimo de humedad. La función del cemento es aglutinar el material y convertirlo en una masa endurecida de carácter estable. El agua hidrata el cemento y ayuda a obtener la máxima densidad lubricando los granos y partículas de suelo. Una vez que el suelo-cemento ha sido mezclado y compactado, se inicia la acción del cemento que, provoca el endurecimiento de la masa.

La combinación ideal del suelo es: 70-80\% de arena, 20 a 30\% de limo, 5 a 10\% de arcilla. Si los suelos son muy arenosos, van a requerir la incorporación de más cemento y a los arcillosos hay que agregarles más arena. Los suelos limosos con un $50 \%$ de arena se estabilizan con un $10 \%$ de cemento.

Las dosis de cemento se calculan como porcentaje en peso del material seco. La humedad de la tierra durante el apisonamiento puede ser del $18 \%$ base húmeda.

\section{Metodología}

\section{Localización}

La granja experimental de la Universidad Surcolombiana se halla situada geográficamente a los $2^{\circ} 5$ ' latitud norte y los $75^{\circ} 20^{\prime}$ latitud oeste, a una elevación de 450 m.s.n.m. en la zona afloran diferentes capas del grupo Honda y de la formación Mesa reportados por varios autores, los cuales atribuyen a la formación Honda espesores entre 1400 y 2600 metros ubicados al oeste de Campoalegre. (Jaramillo, 1983).

De acuerdo con el diagrama para la clasificación de zonas de vida o formaciones vegetales del mundo, por: $\mathrm{L}$.

R. Holdridge; presenta unos parámetros para determinar la zona o zonas de vida del área que se quiere clasificar; los cuales son la biotemperatura anual, precipitación total anual y la relación de evapotranspiración potencial, que separa 120 zonas de vida en el mundo, estos parámetros a su vez corresponde a líneas horizontales y oblicuas, que conforman los hexágonos tridimensional; de acuerdo con esto la Granja experimental de la Universidad Surcolombiana está ubicada en la formación vegetal seco tropical (bs-T), con un promedio anual de lluvias de 1328,4 $\mathrm{mm}$ y biotemperatura de $25,4^{\circ} \mathrm{C}$ y pertenece a la Provincia de Humedad SUB-HUMEDO.

Durante las visitas se realizaron observaciones detalladas de cada una de las partes por donde atraviesan los canales y por donde pasaría la nueva alternativa de conducción, llevando un registro fotográfico de lo observado.

En el trabajo de oficina finalizado el trabajo de campo, se procedió a ordenar, procesar, tabular, calcular, diseñar, analizar y dibujar los resultados obtenidos. Dentro de esta etapa se desarrollaron las siguientes actividades; organización de los datos sobre el estado de la conducción actual para su diagnóstico, diseño de la nueva alternativa de abastecimiento con todas las estructuras que se necesitaban; la compuerta predial, desarenador, aforador, cajillas de inspección, cárcamo y la caseta de bombeo.

Ya en el interior del lote, donde inicia el riego por superficie, se diseñó un tanque disipador de energía para reemplazar el existente, este tanque a su vez servirá de distribución y desarenador. Y se escogieron las tres opciones de revestimiento, la primera sin revestimiento o canales en tierra, que es como actualmente se maneja el riego por superficie en la granja.

También se consideró el revestimiento con suelo cemento, o suelo estabilizado con cemento que es una mezcla en seco de suelo o tierra con determinadas características granulométricas, cemento y en su caso, aditivos.

Y la tercera y última, el concreto de uso común, o convencional, que se produce mediante la mezcla de tres componentes esenciales, cemento, agua y agregados, a los cuales eventualmente se incorpora un cuarto componente que genéricamente se designa como aditivo.

Seguidamente, se realizó el diseño hidráulico de los canales por opciones con las estructuras hidráulicas de medición, control y caída. Culminada esta etapa, teniendo la parte topográfica e hidráulica, se realizó el análisis económico, que consistió básicamente en calcular las cantidades de obra y los precios unitarios, para finalmente saber el presupuesto general por opciones para su comparación. Toda la información que se obtuvo como resultados de los diseños se tabulo para su mejor comprensión a la hora de revisar el documento. 


\section{Resultados}

Se propuso una línea de abastecimiento con una sola pendiente de uno por mil $(0.001=0.1 \%)$, teniendo que demoler las dos primeras alcantarillas, que se imponían en el paso de la tubería enterrada, a $200 \mathrm{~m}$ y $450 \mathrm{~m}$ respectivamente, para que de esta forma la tubería pasará por encima de la cota campana de las nuevas alcantarillas, si obstaculizar la salidas llegando al cárcamo con un profundidad de $2 \mathrm{~m}$. Las alcantarillas propuestas son 6 tubos de 12 ", para remplazar la capacidad del tubo de 24" que está actualmente.

A 40m, se propuso la construcción de un desarenador de 3mx1mx0.7m, diseñado con la metodología de López (1995) y Corcho (1993) seguidamente un aforador Parshall, alcantarillas de inspección de la conducción cada 100m, de $1 \mathrm{x} 1 \mathrm{~m}$ y con la profundidad que lleve la conducción para cada abscisa, entre la cota del terreno (cota negra) y la cota de diseño (cota roja), más $0.3 \mathrm{~m}$ de borde para el paso de la tubería. La tubería llega al cárcamo a $2 \mathrm{~m}$ de profundidad. El cárcamo se diseñó con concreto reforzado de $0.2 \mathrm{~m}$ de espesor, con una profundidad de $3 \mathrm{~m}$ en la parte más baja para darle más capacidad de almacenamiento, con 3mx3m de área en la superficie, la caseta de bombeo quedo similar a la actual y se pretende conectar la tubería que sale del nuevo bombeo con la conducción que viene de la actual caseta que pasa cerca a los salones de la granja.

El tanque disipador de energía quedo con las mismas dimensiones del desarenador $3 \mathrm{mx} 1 \mathrm{mx} 0.7 \mathrm{~m}$, con la misma metodología con la diferencia que en el desarenador, la cajilla de excesos le da la vuelta a este sin pasar por él y llega se une antes del aforador, mientras en el tanque disipador, esta agua de exceso alimenta los dos lagos cerca al tanque con los que cuenta la granja como reservorios.

Las dos opciones de revestimiento suelo cemento y concreto, se diseñaron con un espesor de $5 \mathrm{~cm}$. Todas las estructuras que se colocaron en la conducción incluso en la opción 1, que es para los canales en tierra se propusieron en concreto con un revestimiento de $3 \mathrm{~m}$ aguas arriba y abajo de cada estructura también en concreto para aumentar su vida útil.

Los aforadores diseñados son de dos tipos, Parshall y Ballofet, las estructuras de caída varían en altura según la opción y solo la que se encuentra al comienzo del canal central, se diseñó como estructura de caída escalonada, todas quedaron con un contra vertedero, después del último escalón, para amortiguar el golpe del agua en la caída su ubicación y características se encuentran en tabla 2.y se dejaron unos tramos encofrados para el paso de la maquinaria en el canal oriental, cerca de la derivación con el canal central y en el canal central en la unión con el canal occidental.

El aforo de las canaletas WSC, nos permitió encontrar la curva de afora característica para cada canaleta dando como resultado luego de graficar los resultados una gráfica similar a la figura 1, que corresponde a la curva característica de la canaleta 2. Con la ecuación que se ajusta a la lectura en la reglilla de la canaleta con el caudal que está atravesando la canaleta.

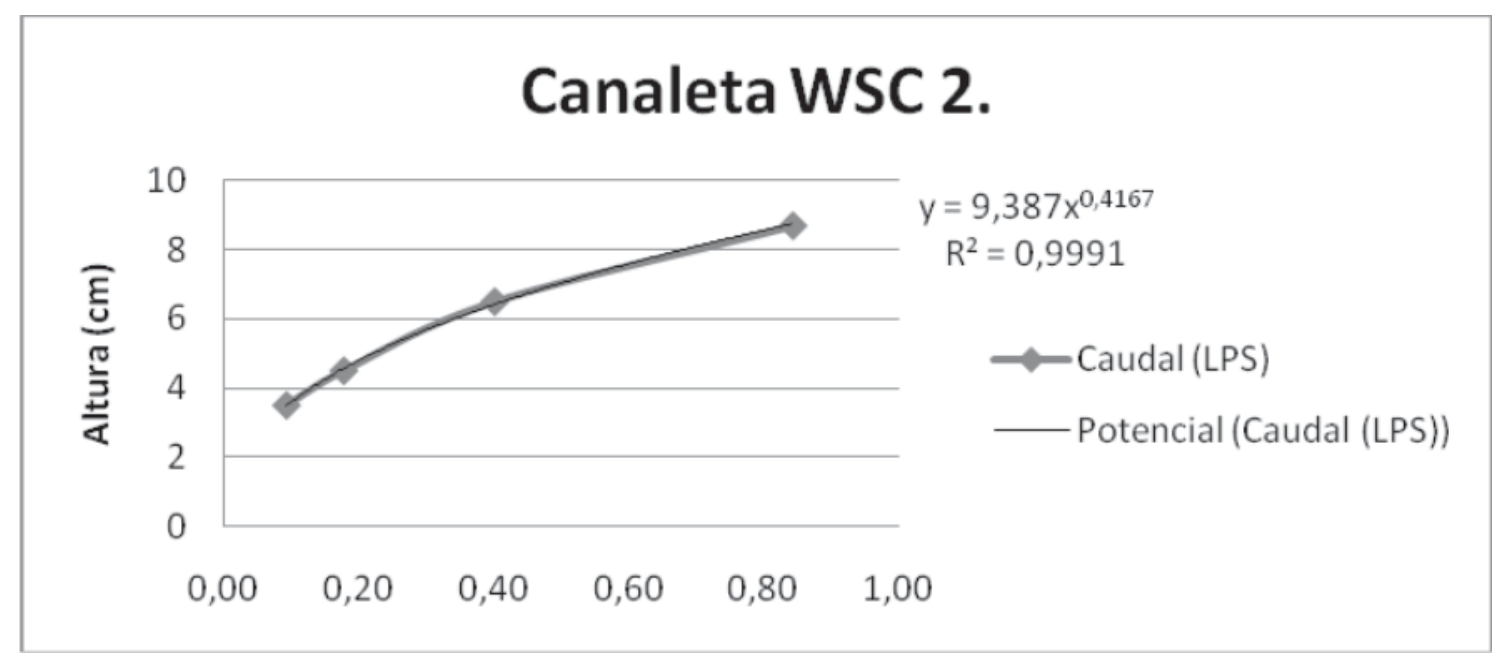

Figura 1. Curva de afora canaleta WSC 2. 
La opción que se recomienda instalar en la granja es independientemente del precio, la opción 3, de revestimiento en concreto, que le dará la eficiencia que necesita el sistema de riego por superficie, con mayor tiempo de durabilidad, menores costos de mantenimiento y con la ingeniería que se necesita se aplique a este centro experimental.

Los movimientos de tierra necesarios para esta opción tabla 1, arrojan como resultado para toda la red de canales, $867.135 \mathrm{~m} 3$ de tierra por corte para la nivelación de los cinco canales con terraplenes en las partes que necesitaban relleno y corte en las partes donde se necesitaba retirar suelo.

Tabla 1. Movimientos de tierra; Corte y relleno. Opción 3.

\begin{tabular}{|c|c|c|c|}
\hline CANALES & CORTE $\left(\mathbf{m}^{\mathbf{3}}\right)$ & RELLENO $\left(\mathbf{m}^{\mathbf{3}}\right)$ & RESULTADO $\left(\mathbf{m}^{\mathbf{3}}\right)$ \\
\hline Oriental & -621.465 & 32.715 & -588.751 \\
\hline Central & -74.835 & 4.217 & -73.556 \\
\hline Occidental & -109.845 & 0 & -109.845 \\
\hline Lagos & -38.485 & 1.57 & -36.911 \\
\hline Triangulo & -58.072 & 0 & -58.072 \\
\hline \multicolumn{2}{|r}{} & TOTAL & -867.135 \\
\hline
\end{tabular}

También se muestra el movimiento de tierra para la realización de los canales después de estar nivelado el terreno, el cálculo de las cantidades de obra para revestir los canales con la mezcla de concreto simple Tabla 2.

Tabla 2. Volumen de tierra para todos los canales. Opción 3.

\begin{tabular}{|c|c|c|c|c|c|c|c|c|c|}
\hline \multirow{2}{*}{ TRAMO } & \multicolumn{2}{|c|}{ FINALES } & \multicolumn{2}{|c|}{ ABCISAS } & & \multirow{2}{*}{ B (m) } & & \multirow{2}{*}{$\begin{array}{c}\text { MOV. } \\
\text { TIERRA }\left(\mathbf{m}^{3}\right)\end{array}$} & \multirow{2}{*}{$\underset{\left(\mathbf{m}^{3}\right)}{\text { CANTIDA DE OBRA }}$} \\
\hline & $\mathbf{h}(\mathbf{m})$ & $\mathbf{b}(\mathbf{m})$ & Inicial & Final & & & & & \\
\hline \multicolumn{10}{|c|}{ CANAL ORIENTAL } \\
\hline TRAMO 1 & 0,18 & 0,2 & 0 & 58 & 58 & 0,56 & 0,06 & 3,9672 & 1,806931103 \\
\hline TRAMO 2 & 0,18 & 0,25 & 58 & 240 & 182 & 0,61 & 0,07 & 14,0868 & 6,125025186 \\
\hline TRAMO 3 & 0,18 & 0,2 & 240 & 420 & 180 & 0,56 & 0,06 & 12,312 & 5,607717217 \\
\hline TRAMO 4 & 0,18 & 0,25 & 420 & 750 & 330 & 0,61 & 0,07 & 25,542 & 11,1058149 \\
\hline \multicolumn{8}{|c|}{ SUB-TOTAL } & 55,908 & 24,6454884 \\
\hline \multicolumn{10}{|c|}{ CANAL CENTRAL } \\
\hline TRAMO 1 & 0,15 & 0,2 & 0 & 262 & 262 & 0,5 & 0,052 & 13,755 & 7,238619957 \\
\hline \multicolumn{8}{|c|}{ SUB-TOTAL } & 13,755 & 7,238619957 \\
\hline \multicolumn{10}{|c|}{ CANAL OCCIDENTAL } \\
\hline TRAMO 1 & 0,2 & 0,2 & 0 & 480 & 480 & 0,6 & 0,08 & 38,4 & 16,08212509 \\
\hline \multicolumn{8}{|c|}{ SUB-TOTAL } & 38,4 & 16,08212509 \\
\hline \multicolumn{10}{|c|}{ CANAL LAGOS } \\
\hline TRAMO 1 & 0,15 & 0,2 & 0 & 257 & 257 & 0,5 & 0,052 & 13,4925 & 7,100478355 \\
\hline \multicolumn{8}{|c|}{ SUB-TOTAL } & 13,4925 & 7,10048 \\
\hline \multicolumn{10}{|c|}{ CANAL TRIANGULO } \\
\hline TRAMO 1 & 0,15 & 0,2 & 0 & 40 & 40 & 0,5 & 0,05 & 2,1 & 1,105132818 \\
\hline TRAMO 2 & 0,15 & 0,2 & 40 & 80 & 40 & 0,5 & 0,05 & 2,1 & 1,105132818 \\
\hline TRAMO 3 & 0,15 & 0,2 & 80 & 120 & 40 & 0,5 & 0,05 & 2,1 & 1,105132818 \\
\hline \multicolumn{8}{|c|}{ SUB-TOTAL } & 6,3 & 3,315398454 \\
\hline & & & & & & \multicolumn{2}{|c|}{ TOTAL } & 127.8555 & 58.375 \\
\hline
\end{tabular}


Esta opción con revestimiento en concreto, requiere un total de mezcla de $58.375 \mathrm{~m} 3$, sin contar el desperdicio. El análisis de precios unitarios para preparar 1metro cubico (m3) de mezcla de concreto simple a todo costo fue de $\$ 129.475$.

Tabla 3. Análisis de costos

\begin{tabular}{|c|c|c|c|c|c|}
\hline No & Opciones & Valor & $\begin{array}{c}\text { Línea de } \\
\text { abastecimiento }\end{array}$ & Valor total & Valor/ha \\
\hline $\mathbf{1}$ & Sin revestir & $\$ 6.959 .108$ & $\$ 42.953 .475$ & $\$ 49.912 .583$ & $\$ 3.327 .305$ \\
\hline $\mathbf{2}$ & Suelo-cemento & $\$ 12.655 .440$ & $\$ 42.953 .475$ & $\$ 55.608 .915$ & $\$ 3.707 .621$ \\
\hline $\mathbf{3}$ & Concreto & $\$ 14.634 .114$ & $\$ 42.953 .475$ & $\$ 57.587 .589$ & $\$ 3.839 .172$ \\
\hline
\end{tabular}

La opción más económica, es la opción sin revestir, debido a que solo se realizará la excavación con las secciones trapezoidales de los canales, la opción 2 de suelo-cemento, que aparentemente era la más aceptable por ser la más económica de los dos tipos de revestimiento, realmente no es la más recomendable por que la diferencia con respecto a la opción 3 no es muy grande, y la diferencia de durabilidad es inferior con respecto a la del concreto.

Los resultados son claros en arrojar que el revestimiento en concreto es la mejor opción, por la vida útil de las estructuras, por la alta eficiencia que le da al sistema, y porque en comparación a la opción alternativa que se planteó en este proyecto es mínima en comparación de los beneficios.

Si los costos se redujeran en un $15 \%$ por concepto de consumo de agua con la adecuación propuesta en el proyecto y la producción se mantenga, la relación beneficio costo sería:

$$
(\mathrm{B} / \mathrm{C})=\frac{7.559 .900}{4.787 .106}=1.58
$$

La tasa interna de retorno (TIR), debe ser igual o mayor al depósito a término fijo $(\mathrm{DTF}=9.73+5)$, siendo la utilidad anual en 5 años igual a la inversión inicial del proyecto. Con tasa de interés del $16 \%$ fijo anual.

\section{Conclusiones}

La opción 1 sin revestimiento, es la más económica en la construcción \$49.912.583 La opción 2. Revestimiento en suelo cemento $\$ 55.608 .915$, la opción 3. Revestimiento con concreto $\$ 57.587 .589$. Para las 15 ha que se cultivan en arroz en la granja. Con un costo por ha para cada opción de \$3.327.305/ha para la opción 1, \$3.707.621/ha y $\$ 3.839 .172 /$ ha para la opción 2 y 3 respectivamente. Los precios no incluyen Administración Impuestos y Utilidades (AIU) ni el $10 \%$ por imprevistos debido al tipo de proyecto.

Los suelos de la granja presentan porcentajes de arena mayores al $72 \%$, por lo tanto son un buen material a utilizarse en la opción de revestimiento con suelo cemento. Solo la serie el caño en la parte final del canal central e inicio del occidental presenta un valor inferior pero no muy retirado al requerido con $69 \%$.

Las pendientes de los canales, en la opción 1 sin revestir son del $0.2 \%$, la opción 2 revestimiento con suelo cemento del $0.3 \%$ y la opción 3 revestimiento con concreto del $0.4 \%$, debido a la diferencia de material para su construcción, las secciones transversales y las velocidades que soportan.

La optimización de la conducción actual, tiene un precio muy inferior al presupuestado para la construcción de la nueva alternativa, con la diferencia, que para este presupuesto de optimización no se tuvo en cuenta el cambio de tubería por encontrarse aparentemente en buen estado, pero, hay que considerar que la vida útil de estas ya ha pasando y pronto habrá que reemplazarla.

Con la nueva alternativa, la tubería del sistema de abastecimiento, no tendrá que pasar por lotes vecinos, el cárcamo y la caseta de bombeo se ubicarán dentro de la granja, pero para ello se debe comprar por lo menos doscientos metros cuadrados $(200 \mathrm{~m} 2)$ del lote que colinda en la parte sur de la granja. La construcción de la nueva alternativa de abastecimiento, aunque sale costosa, independizará el sistema de riego, evitando problemas de vecindad y deterioro de las estructuras por la acción de los descoles de los lotes vecinos. 
Los movimientos de tierra más grandes que se encontraron, fueron en el canal oriental iguales a $588.751 \mathrm{~m} 3$ para la opción 3 de revestimiento en concreto, debido a la longitud del canal, a la topografía que se presenta en el eje que se nivelo para el paso del canal, y por ser esta la opción que presenta mayor pendiente $0.4 \%$. Representando el $67.89 \%$ del movimiento a realizar en esta opción. El otro $32.11 \%$ es el necesario para nivelar los restantes 4 canales que conforman la red de distribución del riego por superficie en la granja.

En general, se puede decir que la cantidad de suelo que se corta se utiliza para hacer los terraplenes, sobrando en algunos canales tierra que se puede adicionar a los suelos de los lotes.

La mezcla de suelo-cemento que es una nueva alternativa de revestimiento, relativamente económica, no muy utilizada puede dar resultado en la granja y puede servir de experimentación para nuevas tesis de grado que se desarrollen. Esta es una opción de revestimiento, que aunque aún no tiene mucho estudio, podría ser una ayuda para los agricultores que tienen riego por superficie para sus cultivos, para que disminuyan las pérdidas que tienen en sus sistemas actuales y a la vez los costos de producción.

\section{Referencias Bibliográficas}

1. Fundamentos de riego. (consultado el 13 Marzo 2009) disponible en: http://www.elriego.com/informa_te/ riego_agricola/fundamentos_riego/riego_superficie.htm.

2. Medición de caudales. (consultado el 07 de Octubre 2009). Disponible en: http://html.medicion. com/medición -de-caudales. HTML.

3. Ojeda O, Adolfo León. 1995. Hidráulica. Conductos con flujo libre canales. Universidad del Cauca. Popayán. Pág. 3-5, 10, 25-32.

4. Pedroza González, Edmundo. 1995. IMTA. Canal Parshall. (consultado el 21 de Octubre2009). Disponible en: http://www.conagua.gob.mx/CONAGUA07/Noticias/canal_parshall.pdf.

5. Lopez, C. Ricardo. 2009. Elementos de Diseños de Acueductos y Alcantarillados. Edit. Escuela Colombiana de Ingeniería, $220 \mathrm{p}$.

6. Corcho R, Fredy H. 1993. Acuedeuctos, Teoría y diseños. Universidad de Medellín.

7. Chamberlain, A. R. 1952. Measuring Water in Small Chanell Whitc WSC Flume. Station circular 200, State College of Washinton.

8.Jarmillo, J. Daniel. 1983. Estudio detallado de suelos del lote “La Granja”. Tesis Ingeniería Agricola Universidad Surcolombiana, 270 . 